\title{
The methane cycle on Titan
}

JONATHAN I. LUNINE AND SUSHIL K. ATREYA

Nature Geoscience 1, 159-164 (2008)

In the main text (not including figure captions), refs $48-54$ are numbered incorrectly. Reference 48 should be replaced with ref. 54 and refs $49,50,51,52,53$ and 54 should be renumbered $48,49,50,51,52$ and 53 , respectively. 